\title{
Nueva Gerencia Pública en la Constitución de la República Bolivariana de Venezuela. Desafíos y perspectivas*
}

\author{
Cuñarro, Edith** \\ Morales, Eduviges***

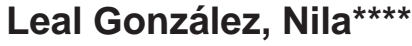

\section{Resumen}

El objetivo de este artículo es analizar la relación entre las bases institucionales del Estado establecidas en la Constitución de la República Bolivariana de Venezuela (1999) y algunos elementos propuestos por la Nueva Gerencia Pública (NGP). La metodología consiste en el estudio crítico de los principales planteamientos de la NGP y su viabilidad en el marco de la Constitución de la República Bolivariana de Venezuela (CRBV), así como el planteamiento de las carencias analíticas que evidencian las corrientes que se engloban bajo la denominación de NGP a la hora de comprender las restricciones democráticas que emanan del sistema político. Los resultados del análisis apuntan a proponer una reforma de los aspectos normativos contemplados en la Constitución de 1999 para que en el desarrollo posterior de su regulación e implementación se flexibilicen los mecanismos de participación de manera que pueda rediseñarse un sistema político que profundice la democracia en Venezuela. Se concluye que, desde esta perspectiva, la constitución de la civilidad tiene que ser investigada en lo que constituye la dimensión de su existencia posible, de los nuevos espacios generados en el presente y por generar en la construcción cotidiana de nuestra polis y de nuestro polemos.

Palabras clave: Estado, Gestión Pública, Constitución de la República Bolivariana de Venezuela, participación ciudadana.

Recibido: 05-01-27. Aceptado: 06-01-10

* Este artículo constituye un avance del Programa de Investigación: Hacia un nuevo modelo político en América Latina: Fortalecimiento y Democratización de la Gestión Pública (financiado por el Consejo de Desarrollo Científico y Humanístico de la Universidad del Zulia (CONDES). Maracaibo-Venezuela).

** Doctora en Ciencias Políticas. Directora del Instituto de Estudios Políticos y Derecho Público. FCJP. LUZ. E-mail: mabelc18 @hotmail.com

*** Doctora en Derecho. Investigadora Adscrita al Instituto de Estudios Políticos y Derecho Público.

**** Doctora en Ciencias Sociales. Investigadora adscrita al Lab. de Antropología. FEC. LUZ. E-mail: nlealg@cantv.net 


\section{New Public Management in the Constitution of the Bolivarian Republic of Venezuela}

Abstract

The objective of this article is to analyze the relationship between the institutional bases of the State established in the Constitution of the Bolivarian Republic of Venezuela (1999) and the elements proposed by New Public Management (NPG). The methodology included the critical study of the principles of NPG and its viability in the framework of the Constitution of the Bolivarian Republic of Venezuela (CBRV), and a study of the proposals of analytical weaknesses that give evidence to what are considered as NPG when analyzing the democratic restrictions that emanate from the political system. The results of the analysis suggest the proposal of a reformation of the normative aspects contemplated in the 1999 Constitution so that in the development of its implementation and mechanisms of participation are made more flexible. Thus a political system can be designed that deepens and solidifies democracy in Venezuela. The conclusion is that from this perspective, the constitution of civic action must be researched in relation to the constitution of the dimension of its possible existence, in relation to the generation of new spaces in the present, and in reference to systems to be generated in the daily construction of our polis, and our polemic.

Key words: State, public management, constitution of the Bolivarian Republic of Venezuela, citizen participation.

\section{Introducción}

La configuración conceptual de la gestión pública contemporánea deriva de la globalización, es el resultado de la estandarización de la administración pública, singular de cada país, bajo un esquema de organización y funcionamiento. Se trata de una guía de implementación, pues su objetivo es establecer reglas a la administración pública, para hacerla más eficaz.

En Venezuela, durante las dos últimas décadas del siglo XX diversos actores políticos le demandaron al sistema político que procesara una serie de reformas que permitieran superar el monopolio representacional instaurado por los dos partidos políticos tradicionales (AD y COPEI) y abriera cauces para la incorporación de la sociedad a la formulación, evaluación y control de las políticas públicas. Estas reformas fueron, en su mayoría, bloqueadas en el parlamento por los mismos partidos del status, situación que llevó a una turbulencia que culminó, al menos en algunos de sus aspectos, en el proceso constituyente de 1999 que elaboró una nueva carta fundamental en la cual se consagra "la esencia participativa del Estado".

En una dinámica en la cual el Estado busca desligarse de algunas cargas y la sociedad demanda incorporarse al proceso decisional, se estableció un marco normativo que tiende a facilitar la instauración de una matriz sociocéntrica que viabilice la corresponsabilidad. En el ámbito de la Administración Pública, esta matriz implica la incorporación de la ciudadanía activa en el control de las políticas públicas. Es en ese sentido que se sostiene que las bases institucionales del Estado establecidas en la Constitución de la República Bolivariana de Venezuela (CRBV) de 1999 hacen posible que se incorporen nuevos mecanismos que democraticen la gestión pública. 
Nueva Gerencia Pública en la Constitución de la República Bolivariana de Venezuela Cuñarro, Edith; Morales, Eduviges y Leal González, Nila

\section{Las transformaciones de la relación Estado-sociedad}

Los cambios en la relación Estadosociedad aparejan cambios fenoménicos y paradigmáticos en la Administración Pública. Diferentes corrientes interpretan y promueven modelos de gestión pública que, se supone, adecuan la gestión pública a las nuevas realidades. Por un lado, las corrientes que se engloban bajo la común denominación de NGP, proponen una serie de cambios que buscan incorporar a la sociedad al control de la gestión pública, propuesta que, en general, se comparte como progresiva en la búsqueda de una mayor corresponsabilidad y transparencia. Pero, además, estas tendencias, sostienen la necesaria neutralidad de la administración frente a la política. Dicho lineamiento se considera inviable pues la gestión siempre se encontrará teñida por los matices que le impriman las decisiones políticas, en ese sentido, se considera necesario aclarar, la importancia del vínculo entre política y Administración pública.

El moderno Estado de Bienestar o asistencial tuvo su origen en el compromiso político entre los principios de mercado y las exigencias de la justicia social del movimiento obrero europeo. Agotado el período del denominado "fordismo atlántico" y avanzada la fase de la globalización que se inició en 1989, la relación de la entidad estatal con la sociedad se ha visto también modificada.

El nuevo Estado Social que está emergiendo es una respuesta a la nueva problemática. En ese sentido, Bresser (2004) señala que ya no se trata del Estado ultraliberal, con el que sueña la nueva derecha, un Estado mínimo que podría garantizar contratos y derechos de propiedad. Ni siquiera es más pequeño que el viejo Estado Social Democrático, si medimos el tamaño del Estado por la carga impositiva: es decir, por la relación entre ingreso y producto interno bruto.

En el nuevo Estado el tamaño no tiende a disminuir, sino por el contrario, tiende a aumentar moderadamente en la medida en que los costos en educación y en salud-que permanecen bajo su responsabilidad, tienden a incrementarse junto con el costo de los demás bienes y servicios. El autor señala que se acabó la ola ultraliberal que se inició a fines de la década de 1970, como se evidencia de la alternancia en el poder de las coaliciones políticas de derecha o de izquierda, la cuales, se supone, continuarán definiendo las democracias en el futuro cercano.

Bresser propone denominar a la entidad estatal que se está configurando en los albores del siglo XXI, como Estado Social Liberal. Explica que el compromiso de la sociedad con los derechos sociales de las personas se mantiene y acota que, fundamentalmente, la diferencia está dada por el hecho de que el nuevo estado descansa en el mercado o en la competencia administrada mucho más que en lo que el Estado Social Democrático ha hecho. Además, el Estado Social Liberal cree en la competencia, la cual no es percibida como lo contrario de la cooperación.

Esta fe en los mercados y en la competencia se expresa, para el autor, de dos maneras: En primer lugar, en el rechazo a la idea de un Estado productor de bienes y servicios para el mercado. El apoyo a la privatización de las empresas estatales que son competitivas promueve justamente dicha creencia. En segundo lugar, 
se expresa en la afirmación de que actividades que no son necesariamente exclusividad del Estado, tales como los servicios de carácter social y científico y que no son esencialmente monopólicos, no tienen porque ser realizados directamente por el Estado. Estos servicios deben ser financiados por el Estado, pero pueden ser ejecutados competitivamente por organizaciones públicas no estatales o sin fines de lucro.

En el marco de estos cambios impulsados por la globalización, la relación Estado-Sociedad ha sido enfocada desde diversas perspectivas. Por un lado, se encuentran, aquellas miradas, realizadas, fundamentalmente, desde los países centrales, que se han denominado bajo la sigla común de NPG.

La crisis del sistema financiero mundial de los 80 y su particular expresión en los sistemas e instituciones políticas nacionales, así como la rearticulación de las relaciones Estado/sociedad que comienza a manifestarse en muchos países bajo la impronta de la liberalización de las eco- nomías y la erradicación de la matriz Estadocéntrica, fueron factores determinantes en el diseño y las prácticas de gestión de las administraciones públicas sustentado en la doctrina del llamado New Public Management o Nueva Gestión Pública (NGP) (Quintin, 2002: 116).

El término gestión ha tenido un empleo muy intenso en Francia desde tiempo atrás. Nació con la ciencia de la administración pública misma: cuando se hizo referencia a la ejecución de las leyes como un asunto necesario a la "gestion des affaires publiques" (Guerrero, 2001:3). En español dicho vocablo ha sido usado como sinónimo de administración, o, más generalmente, como una parte de la misma. Sin embargo, desde la década de los ochenta, gestión comenzó a ser usada como antónimo de administración, precediendo e inspirando a la corriente anglosajona de la nueva gestión pública (new public management) ${ }^{1}$ (Guerrero, 2001: 4). En el seno de estas tendencias se discute la relación existente entre el sector público y el

1 Los nuevos modelos de gestión pública aparecieron inicialmente en el Reino Unido, y una vez aterrizados en suelo estadounidense, australiano y neozelandés, han tenido un desarrollo propiamente anglosajón. El aporte primigenio en esta línea conceptual, se debe al administrativista francés Michel Messenet. Su obra La Nouvelle Gestion Publique: pour un Etat sans Burocratie (La Nueva Gestión Pública: por un Estado sin Burocracia), es posiblemente la exposición original de la nueva gestión pública. Esta obra, publicada en 1975, plantea el carácter "nuevo" de la gestión pública y usa extensivamente el término management, alternado con el vocablo gestión, toda vez que se proclama un sentimiento anti-burocrático como asunto central de su ideario. Las ideas de Messenet están inspiradas fundamentalmente en la administración privada, que concebía ésta de una manera distinta a la tradición fundada por Henri Fayol. Octave Gelinier, quien prologó su libro, usa el término "management public" para referirse a una técnica que refleja con nitidez tiempos emergentes, particularmente la innovación y la anticipación, así como la aptitud para "gerenciar (gérer) el cambio". Gelinier plantea una tesis medular de la neo-gestión pública: plantea que ha dejado de estar centrada en la "sociedad política, mundo del voto y del control de los ciudadanos", y ha pasado a la sociedad civil, cosmos del contrato, del ejercicio de la libertad creativa de las personas (Guerrero, 2001:2). 
Nueva Gerencia Pública en la Constitución de la República Bolivariana de Venezuela Cuñarro, Edith; Morales, Eduviges y Leal González, Nila

privado. Aunque este encuentro significa un caudal de riqueza para la nueva disciplina, también lleva dentro de sí grandes desafíos para la conjunción de culturas administrativas diversas.

Tradicionalmente el management ha consistido en la aplicación del análisis a sistemas de la empresa, por igual al sector privado y al público. Pero, con el tiempo, el management public se ha ido basando en la aplicación del marketing al sector público, aunque por principio las lógicas de la administración pública y el marketing sean opuestas: la administración pública es normativa y porta la idea del bien público, se basa en la ética y busca la satisfacción del interés común, en tanto que el marketing busca satisfacer el bienestar individual, es oportunista y se basa en la novedad. La administración pública representa la situación permanente del Estado y tiene responsabilidades a largo plazo, mientras que el marketing está atento al mercado en un momento determinado de su funcionamiento y tiene objetivos a corto plazo. La primera se basa en la lógica política y la segunda, en la lógica económica ${ }^{2}$. Teniendo conciencia de estas particularidades, en este trabajo se pone el acento en los elemen- tos de la NPG que se relacionan con las bases institucionales del Estado (especialmente las referentes a su esencia participativa) establecidas en la Constitución de la República Bolivariana de Venezuela y que norman la contraloría social, la coresponsabilidad y la transparencia.

\section{Las "restricciones democráticas" en la Administración Pública}

Pero, por otro lado, las teorías de la NGP, tienen, todas ellas una carencia muy importante, no superan la dicotomía política-administración, que los cambios impulsados por la globalización han llevado a que sea reconceptualizada.

La globalización está logrando que los Estados-nación sean cada vez más interdependientes, está fortaleciendo el mercado de bienes y servicios, de capitales y tecnologías. Todos los días los mercados inundan nuevos sectores de la economía y adquieren mayor influenica sobre los sectores que ya constituían su ámbito de acción. Pero ello no implica que el ámbito político pierda poder o que las decisiones políticas pierdan relevancia. Por el contrario, en la medida que tanto la sociedad como el mercado se hacen

2 El modelo neo-gestionario contemporáneo, no constituye un tronco del cual emanan ramas especializadas, sino un tallo relativamente corpóreo alimentado por raíces fuertemente hermanadas. Dicho tallo tiene la denominación general de nueva gestión pública (new public management), que en la modalidad británica se llama modelo Whitehall. Bajo la variante estadounidense se conoce como gobierno empresarial y esquema post-burocrático. Concebido en la escala global, constituye el modelo OCDE (Organización de Cooperación para el Desarrollo Económico). Cada una de estas modalidades comparten las líneas generales de la nueva gestión pública, pero ponen énfasis en algunos aspectos de conformidad con su cultura nacional o su espectro internacional: las versiones estadounidenses subrayan los atributos empresariales bajo la noción del gobierno emprendedor y los rasgos procesales, con el modelo posburocrático, en tanto que la OCDE ofrece la noción primigenia de la orientación hacia el cliente (Guerrero, 2001: 4). 
cada día más complejos y la sociedad civil cada vez más exigente y capaz de ejercer un efectivo control social, se incrementa al mismo tiempo el carácter estratégico de las decisiones políticas y la necesidad de que sean tomadas por funcionarios de gobierno que estén dotados de mayor autonomía.

Tradicionalmente se pensaba en los funcionarios públicos de alto nivel como burócratas o técnicos. Actualmente, en cambio, la idea de un funcionario burócrata y neutral que se limita a aplicar la ley o que sigue estrictamente las políticas definidas por los políticos elegidos -una idea que era central en la administración pública burocrática- tiende a ser desplazada paulatinamente. La idea de un servicio público de naturaleza a-política cada día se hace más difícil de ser sostenida (Bresser, 2004: 15).

Entre los funcionarios aún podemos distinguir los políticos elegidos de los funcionarios públicos de alto nivel; sin embargo, todos son políticos, todos establecen políticas y participan directamente en el diseño y la operación de las instituciones políticas.

En la medida en que su rol como funcionarios cambia deberán sustituir la clasica ética burocrática de la disciplina por la ética de la responsabilidad. Se espera de ellos que sean responsables ante la sociedad y es así como su rol deja de ser formalmente técnico y pasa a ser un rol político. En el caso de Venezuela, estas características se acentúan, por su carácter de Estado petrolero. Estas especificidades del caso venezolano, deben ser tomadas en cuenta, a los efectos de la relación entre las bases institucionales del Estado establecidas en la Constitución de 1999 y su relación con algunos elementos de la NGP.
Respecto a la separación entre administración pública y política, seguimos los argumentos expuestos por Bresser (2004), en lo que se refiere a las "restricciones democráticas". El explica que, en la mayoría de los casos, los científicos políticos y consultores que trabajan en este campo tienden a suponer que la reforma de la Administración Pública implica dotar a la organización del Estado de una perfeccionada racionalidad; y a partir de esta idea concluyen que, al elegir opciones, los funcionarios deberían utilizar el criterio económico o de eficiencia como el parámetro de referencia principal (Bresser, 2004:32).

Más, para el autor, el error de este enfoque radica en que, en términos de la práctica existente en las democracias modernas, la racionalidad instrumental -y el subsiguiente criterio económico- son, sin duda, importantes; pero en un sistema democrático hay un criterio previo y de mayor peso: responder a los requerimientos reales de los electores (a este constreñimiento de las decisiones políticas por los requerimientos de las mayorías electorales se le denomina "restricción democrática"). La restricción democrática deberá prevalecer toda vez que su carácter particular viene dado por la acción colectiva a través del Estado. No es suficiente que las decisiones sean racionales, las decisiones también deben ser democráticas, lo que significa que deben responder a los requerimientos reales de los electores (Bresser, 2004:33).

A cada tipo de restricción corresponde una lógica diferente. Mientras que la racionalidad instrumental domina en lo que respecta a las exigencias económicas, el debate público y la construcción de consensos constituyen los elementos clave cuando se trata de las exigencias políticas. 
Nueva Gerencia Pública en la Constitución de la República Bolivariana de Venezuela Cuñarro, Edith; Morales, Eduviges y Leal González, Nila

Se supone, generalmente, que la restricción económica es la única legítima, porque la perciben como la perspectiva "racional" para tomar decisiones. De esta manera, entienden la influencia política sobre las decisiones públicas, no como una exigencia o una restricción, sino como un obstáculo. De acuerdo con esta perspectiva, los políticos siempre serán "populistas", atentos sólo a sus propios intereses. En lugar de actuar de acuerdo con lo que es "racional", siempre estarán rindiendo pleitesía a electores mal informados, mientras responden a las presiones de los grupos de interés. Esta dificultad para comprender la restricción democrática es el resultado del carácter que ha tenido la democracia en su historia reciente. El derecho universal a elegir y ser elegido es un fenómeno del siglo XX. Cuando el régimen político es democrático, la restricción de orden político adquiere un carácter decisivo, desde el momento en que constituye la fuente esencial de legitimidad. En democracia, el proceso político puede ser estorbado por los intereses individuales o por el peligro latente de una acción colectiva; pero, como contrapartida, cuando se logra neutralizar relativamente los intereses individuales, la democracia conduce a decisiones más competentes en la medida en que se originen en un amplio debate público (Bresser, 2004:35).

\section{La Administración Pública en la Constitución de la República Bolivariana de Venezuela (1999)}

En el caso de Venezuela, estas particularidades se acentúan por ser el Estado venezolano un Estado petrole- ro. De acuerdo a los criterios antes sostenidos, se procede a establecer en este aparte, la relación entre algunos elementos de la NGP y las bases institucionales del Estado instituidas en la Constitución de la República Bolivariana de Venezuela de 1999, en lo que atañe a: previsión de controles sociales y disposiciones sobre participación ciudadana en la gestión pública; previsión para el desarrollo de la modalidad de gestión por resultados que sirva de base para la asignación presupuestaria; previsión de principios, entre los que destacan los de transparencia y responsabilidad, que orientan el régimen socioeconómico, la gestión fiscal, la organización y el funcionamiento del gobierno y de la administración pública (Cuñarro, 2004).

Los medios que la Constitución de 1999 ofrece para orientar las "rupturas" administrativas en esa dirección son, entre otros:

- Previsión de controles sociales y disposiciones sobre participación ciudadana en la gestión pública (artículos $62,70,71,72$ y 143), contribuyendo a ampliar y fortalecer los dispositivos de responsabilización de la administración pública.

- Previsión para el desarrollo de la modalidad de gestión por resultados que sirva de base para la asignación presupuestaria. El artículo 315 establece que en el presupuesto anual de gastos se establecerá, para cada crédito presupuestario, el objetivo específico a que esté dirigido, los resultados concretos que se espera obtener, y los funcionarios públicos responsables por el logro de tales resultados. 
- Previsión de principios, entre los que destacan los de transparencia y responsabilidad, que orientan el régimen socioeconómico (artículo 299), la gestión fiscal (artículo 311), la organización y el funcionamiento del gobierno (artículo 6) y de la administración pública (artículo 141). Respecto a esta última, se señalan los principios de honestidad, participación, celeridad, eficacia, eficiencia, transparencia, rendición de cuentas y responsabilidad en el ejercicio de la función pública (Asamblea Nacional Constituyente, 1999).

Los principios antes señalados, deben ser implementados en la Administración Pública para que los lineamientos constitucionales se materialicen en la cotidianidad administrativa, ya que ellos inducen procesos de evaluación del desempeño institucional, sustentados en indicadores de gestión, con el propósito de hacer efectiva la responsabilidad pública y la transparencia.

Los indicadores de gestión se construyen en el marco de un proceso de planificación estratégica institucional. Tienen como objetivo la construcción de un sistema de medición y evaluación de la gestión de programas, proyectos y servicios públicos de forma que permitan:

a) Proporcionar información regular a los ministerios, comunidades afectadas y al público en general sobre el uso de los recursos y la calidad de la gestión.

b) Incorporar información relacionada con el desempeño de las instituciones en la discusión presupuestaria.

c) Generar bases sustentables de información para la toma de decisiones de los organismos, impulsando una gestión comprometida con los resultados.
La construcción de los indicadores de gestión debe cumplir un conjunto de requisitos con el fin de garantizar el éxito en su aplicación, entre los cuales se destacan los de pertinencia, independencia, economía, confiabilidad, publicidad, elaboración participativa, simplicidad y comprehensividad. Pero el requisito más importante es la capacidad técnica de los funcionarios y la disposición de la gerencia para llevar adelante y sostener un proceso de planificación estratégica (Quintín, 2002: 117).

Los "compromisos de resultados" deberán establecerse a través de contratos celebrados entre los órganos responsables de formular las políticas públicas y asignar los recursos financieros, y las entidades responsables de producir o suministrar un servicio público (cualquiera sea su naturaleza jurídica) y que requieran de financiamiento estatal total o parcialmente. Por otra parte, la "evaluación del desempeño" es un proceso sistemático de análisis de los resultados alcanzados en un período por un programa, considerando los propósitos que lo justifican, el grado de cobertura, la eficiencia, la oportunidad y el autofinanciamiento, y el tipo de indicadores de gestión empleados (Aguilar, 2000:15).

Los resultados de la evaluación del desempeño serán informados por parte del órgano rector del sistema tanto a los miembros de los poderes Ejecutivo y Legislativo como a los ciudadanos, sin perjuicio del ejercicio de las formas clásicas o tradicionales de rendición de cuentas.

La construcción de la viabilidad a la democrática de la Administración Pública, no sólo exige romper con los viejos mitos que ahora se renuevan en las diversas corrientes asociadas con la administración, sino que requiere dotar a la ciudadanía de 
Nueva Gerencia Pública en la Constitución de la República Bolivariana de Venezuela Cuñarro, Edith; Morales, Eduviges y Leal González, Nila

auténticos medios de influencia sobre la Administración Pública, que además de permitir un re-equilibrio del poder (más democracia) aseguren un mejor control y más eficiencia de la administración.

Aunque la participación ciudadana en los procesos de formación de las decisiones públicas es uno de los medios tradicionales, no siempre asegura estas tres condiciones: democracia, eficiencia y control, que justifican la reforma política en la Administración Pública. Para poner la administración pública al servicio de la ciudadanía muchas reformas son necesarias, una de las cuales es el perfeccionamiento de los sistemas de control, a fin de evitar la privatización de las decisiones públicas, que es el mayor peligro que acompaña a la implementación de los parámetros de la NGP. A tal efecto es indudable que son imprescindibles, tanto una reforma política que democratice el sistema político, como una reforma administrativa que profesionalice y flexibilice la función pública. Para construirle viabilidad a estos cambios, y a la vez para propender a un equilibrio de poderes cabe adoptar como estrategia la administración de la gestión pública convirtiendo a la ciudadanía en un sujeto directo de su control.

Como manera de darle basamento jurídico a la democracia participativa, la Constitución de 1999 mantuvo y amplió el carácter social del Estado. Esta ampliación que apareja un mayor grado de intervención del Estado, tiene como consecuencia el establecimiento de mayores instancias de control del aparato público sobre la sociedad.

La democratización de la Administración Pública obliga a dotar de auténticos medios de influencia a la ciudadanía. Para esto son necesarias, por lo menos dos cuestiones. Primero, es preciso ampliar la perspectiva que habitualmente se tiene sobre los posibles medios de control. La participación ciudadana en los procesos de formación de las decisiones públicas -o representación social- es el medio por excelencia. Pero también la elección de los servicios y la coproducción pública pueden constituirse en formas de control ciudadano sobre la administración pública (Cunill, 2004: 44). Segundo, hay que considerar, que no hay posibilidades de propender a una significativa democratización de la Administración Pública, si la ciudadanía no puede constituirse como actor político que equilibre el poder de la burocracia y la controle eficientemente, tratando de fortalecer las consideraciones de equidad en sus decisiones. Pero además, no hay real democratización de la Administración si en el intento resulta lesionada su capacidad de producir bienes y servicios de calidad para todos.

La transparencia ha sido destacada como uno de los criterios más básicos, ya que no siempre la ciudadanía concernida puede actuar (directamente) en forma crítica y activa, así como no toda la Administración Pública puede ser objeto de la influencia ciudadana directa. De hecho, el desafío es crear todas las condiciones básicas de la democratización de la administración, de forma de que pueda operar un re-equilibrio de poder a favor de la ciudadanía que devenga en mejores servicios y regulaciones públicas para que todos puedan construir el bienestar colectivo (Cunill, 2004: 45).

La extensión del Estado Social se evidencia en los artículos comprendidos entre el 19 y el 129 de la Constitución, los 
cuales definen la existencia de una "Carta de Derechos" del ciudadano, que comprende la existencia de los llamados derechos humanos, los derechos sociales y de las familias, derechos culturales, derechos indígenas y derechos educativos.

Por otro lado, el texto constitucional consagra el derecho de los ciudadanos a participar en los asuntos públicos, directamente o por medio de sus representantes, y se estipula que el Estado tendrá la obligación de facilitar las condiciones más favorables para su práctica (art. 62). En el artículo 70 se definen los medios de participación en lo político, en lo social y en lo económico (las instancias de atención ciudadana, la autogestión, la cogestión, las cooperativas, las empresas comunitarias y demás formas asociativas guiadas por la cooperación y la solidaridad) (Leal, 2003).

En suma, las expresiones del Estado Social de Derecho en la Constitución de 1999 significan el fortalecimiento de la centralidad estatal, mientras, por otra parte, se desarrollan también los canales de participación ciudadana en los asuntos públicos. La superposición de ambos lineamientos constitucionales, por un lado el carácter interventor del Estado, y por el otro, la aspiración establecida en la normativa de abrir espacios a la participación ciudadana en la formulación, implementación y control de las políticas públicas, parecen a simple vista contradictorias. ¿Cómo delegar funciones y descentralizar recursos manteniendo la centralidad del Estado, como su carácter "social" lo prefigura? Se podrá caracterizar el proceso de reconfiguración del Estado venezolano como una tendencia a constituirse en un Estado Social Liberal? Este es uno de las grandes interrogantes a los que se enfrenta en Venezuela la posibilidad de establecer una matriz sociocéntrica para armonizar las relaciones entre el Estado y la sociedad.

\section{Conclusiones}

La existencia de una Administración Pública cooptada por intereses políticopartidarios y particulares es lo que, en su momento, se intentó combatir a través del modelo burocrático. Su ideal fue un cuerpo de funcionarios altamente profesionalizado que sirve con eficiencia a la política y que es democráticamente controlado por ella. Las nociones de discrecionalidad, responsabilidaad y resultados reemplazan a las tradicionales de apego a la jerarquía y a las normas, pero el postulado es el mismo: un cuerpo administrativo profesionalizado. La existencia de un servicio público profesional es asumida como una premisa del buen gobierno, sin la cual es impensable una relajación de los controles. En un país que, como Venezuela, ha predominado el clientelismo y el corporativismo, se hace difícil concebir la instauración de la profesionalización en la Administración Pública, no obstante, algunos de sus elementos como el control social, la transparencia y la responsabilidad son recomendables para su mejor administración.

La NGP surge en un contexto de Estado de Bienestar creado para ofrecer a la población "minimos de bienestar", en tanto que en Venezuela se batalla para ofrecer "mínimos de subsistencia". Por tanto, la creación, desarrollo y aplicación de una disciplina de la gestión pública, en vez de atender sólo temas como eficiencia en servicios básicos se debe preocupar por su cobertura, por problemas de legitimidad, crisis institucional, corrupción, modernización de las 
Nueva Gerencia Pública en la Constitución de la República Bolivariana de Venezuela Cuñarro, Edith; Morales, Eduviges y Leal González, Nila

dependencias gubernamentales, además de atender a la interacción con la esfera política y buscar el equilibrio entre administración pública y democracia.

La democracia no es un estadio sino una tendencia que se construye día a día. En Venezuela se vive actualmente un proceso de fractura, pero este proceso puede permitirnos avanzar en la tendencia hacia una profundización de la democracia. La presencia y actuación beligerante de redes, circuitos y actores de la sociedad en la actual coyuntura política venezolana, son los referentes empíricos que permiten albergar esperanzas. Los venezolanos abandonamos la anomia y empezamos a atrevernos a participar activamente en la ejecución de nuestro proyecto político.

La utopía de la democracia total no es una realidad humanamente alcanzable, pero lo que vale es la tendencia a alcanzarla y, sobre todo, la convicción que la democracia no puede ser limitada, restringida, otorgada. O es proceso hacia la construcción de la sociedad civil, o es el vacío sustituido por lo imaginario en tanto justificación de la desigualdad y la violencia. La democracia es un camino interminable pero necesario de recorrer si el hombre sigue fiel a su elección de libertad como ser social. Es un proyecto no concluido, cuyos avances y retrocesos deben ser detectados en su significación en cada momento del presente. Desde esta perspectiva, la constitución de la civilidad tiene que ser investigada en lo que constituye la dimensión de su existencia posible, de los nuevos espacios generados en el presente y por generar en la construcción cotidiana de nuestra polis y de nuestro polemos. El reto de la construcción de un nuevo modelo político pone en evidencia la necesidad de reflexionar acerca de los aspectos normativos señalados en la Constitución de 1999 de manera que pueda rediseñarse un sistema político que profundice la democracia en Venezuela.

\section{Referencias Bibliográficas}

Aguilar, Luis F. (2002). Seminario "Política Pública y NGP. La Dirección Estratégica". Facultad de Ciencias Económicas y Sociales, Universidad del Zulia.

Asamblea Nacional Constituyente (1999). Constitución de la República Bolivariana de Venezuela. Caracas.

Bresser-Pereira, Luiz C. (2004). "La restricción económica y la democracia", en Política y Gestión Pública, Editorial Fondo de Cultura Económica- CLAD, pp. 13-42, Argentina.

Cunill, Nuria (2004). "La democratización de la Administración Pública. Los mitos a vencer", en Política y Gestión Pública, Editorial Fondo de Cultura Económica- CLAD, pp. 43-90, Argentina.

Cuñarro, Edith (2004). "Modernización Reflexiva y Reforma del Estado", en Revista Opción No. 43, FEC-LUZ, Maracaibo.

Guerrero, Omar (2001). Nuevos modelos de gestión pública, www. Revista. Unam.mx/vol.2/num3/art3/ consultada el 15 de mayo de 2004.

Leal, Nila (2003). "Capital social y nuevas formas de ciudadanía", en Revista Cuestiones Políticas No. 33. Instituto de Estudios Políticos y Derecho Público. FCJP-LUZ, Maracaibo, Venezuela.

Quintín, Antonio (2002). Patrimonialismo, burocracia y posburocracia en el diseño institucional de la administración pública venezolana, Mimeo, Universidad Central de Venezuela, Caracas. 